\title{
ASSOCIATION OF ANDROGENETIC ALOPECIA WITH METABOLIC SYNDROME
}

\author{
P. Anila Sunanandini Nayar ${ }^{1}$, G. Suryanarayana ${ }^{2}$, Sameera Boda ${ }^{3}$, Hanusha Kota ${ }^{4}$, Subhashini Konala ${ }^{5}$, Chakravarthy S.6, Sruti Sree , \\ Vindya ${ }^{8}$
}

1 Professor, Department of DVL, Andhra Medical College, King George Hospital, Vishakhapatnam, Andhra Pradesh, India. ${ }^{2}$ Associate Professor, Department of DVL, Andhra Medical College, King George Hospital, Vishakhapatnam, Andhra Pradesh, India. ${ }^{3}$ Postgraduate Student, Department of DVL, Andhra Medical College, King George Hospital, Vishakhapatnam, Andhra Pradesh, India. ${ }^{4}$ Postgraduate Student, Department of DVL, Andhra Medical College, King George Hospital, Vishakhapatnam, Andhra Pradesh, India. ${ }_{5}^{5}$ Senior Resident, Department of DVL, Andhra Medical College, King George Hospital, Vishakhapatnam, Andhra Pradesh, India. ${ }^{6}$ Postgraduate Student, Department of DVL, Andhra Medical College, King George Hospital, Vishakhapatnam, Andhra Pradesh, India. ${ }^{7}$ Postgraduate Student, Department of DVL, Andhra Medical College, King George Hospital, Vishakhapatnam, Andhra Pradesh, India. ${ }^{8}$ Postgraduate Student, Department of DVL, Andhra Medical College, King George Hospital, Vishakhapatnam, Andhra Pradesh, India.

\section{ABSTRACT}

\section{BACKGROUND}

Androgenetic alopecia has been associated with increased risk of coronary heart disease in various studies. Androgenetic alopecia is the most common type of alopecia in men., characterised by the transformation of thick terminal hair follicles in to thin vellus like hair follicles. It occurs under the influence of androgens in genetically predisposed individuals. The mode of inheritance is polygenic. The relationship between androgenetic alopecia and metabolic syndrome, is a known risk factor for atherosclerotic diseases. Metabolic syndrome is a cluster of inter related risk factors that increase the risk of atherosclerotic cardiovascular disease. Our study looked at the association between metabolic syndrome and androgenetic alopecia in the age group of $20-45 \mathrm{yrs}$. men. We wanted to study the association between androgenetic alopecia and metabolic syndrome.

\section{METHODS}

A descriptive cross-sectional study was done on men in the age group of 20 to 45 years. Two groups are taken in to the study. The first group is androgenetic alopecia group and the second group is non alopecia group. Study is undertaken for a period of 3 months, hence feasible sample size 30 is taken. Age of the study population is restricted to 20-45 years because incidence of androgenetic alopecia increases with age. Statistical association between androgenetic alopecia and metabolic syndrome was studied using students $t$ test and chi square test SPSS version 20 .

\section{RESULTS}

Metabolic syndrome was seen in 8 out of 30 samples (26\%) in the androgenetic alopecia group, 4 out of 30 samples (13\%) in nonalopecia group, $\mathrm{p}$ value is 0.015 . Central obesity and HDL are elevated in most of the samples of androgenetic alopecia group. Limitation of our study was the small sample size.

\section{CONCLUSIONS}

High prevalence of metabolic syndrome is seen in men with androgenetic alopecia. Investigating for metabolic syndrome in patients with androgenetic alopecia is helpful for early detection and prevention of coronary artery disease.

\section{KEY WORDS}

Androgenetic Alopecia, Metabolic Syndrome, Obesity, High Density Lipoproteins

HOW TO CITE THIS ARTICLE: Nayar PAS, Suryanarayana G, Boda S, et al. Association of androgenetic alopecia with metabolic syndrome. J. Evolution Med. Dent. Sci. 2019;8(30):2403-2406, DOI: 10.14260/jemds/2019/526

\section{BACKGROUND}

The term pattern hair loss or common balding or androgenetic alopecia is a form of hair loss that occurs in a generally distinctive pattern and characterised by a progressive decline in hair fibre production by scalp hair follicles and their eventual miniaturisation. There are studies that show association between androgenic alopecia and metabolic syndrome.

'Financial or Other Competing Interest': None.

Submission 02-12-2018, Peer Review 11-07-2019,

Acceptance 17-07-2019, Published 29-07-2019.

Corresponding Author:

Dr. Sameera Boda,

Room No. 24, PG Women's Hostel,

KGH, Maharanipeta, Vishakapatnam,

Andhra Pradesh, India.

E-mail: sameeraboda90@gmail.com

DOI: $10.14260 /$ jemds $/ 2019 / 526$
This study is done to check the association between androgenetic alopecia and metabolic syndrome.

\section{Study Type \\ Descriptive cross-sectional study.}

\section{Inclusion Criteria}

Androgenic Alopecia Group- Men in the age group of 20-45 years with Norwood Grade 3 and above are included in the androgenetic alopecia group.

Non-Alopecia Group- Men in the age group of 20 -45 years with dermatological disease other than androgenetic alopecia are taken in to non-alopecia group.

\section{Exclusion Criteria}

Patients receiving hormonal treatment with testosterone, those on corticosteroid and those with hypothyroidism and psoriasis were excluded. 


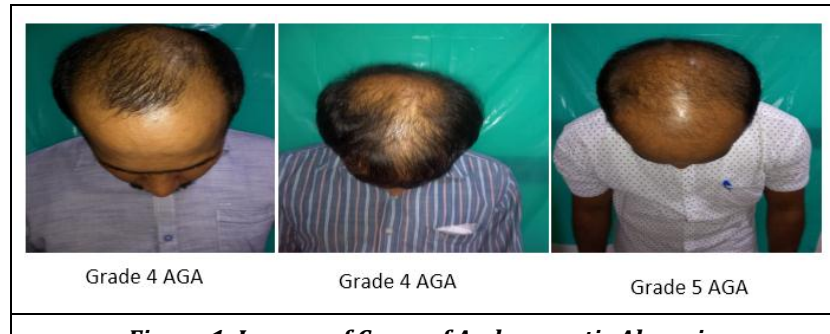

Figure 1. Images of Cases of Androgenetic Alopecia

\begin{tabular}{|c|c|c|c|}
\hline & AGA Group (30) & Non-Alopecia (30) & p Value \\
\hline Age (years) & $35.9(\mathrm{n})$ & $42(\mathrm{n})$ & 0.003 \\
\hline Waist circumference (cms) & $132(\mathrm{n})$ & $91.6(\mathrm{n})$ & 0.101 \\
\hline Smoking & 12 & 10 & 0.121 \\
\hline Alcohol & 14 & 10 & 0.443 \\
\hline \multicolumn{3}{|c|}{ Table 1. Descriptive Characters of Study } \\
\hline
\end{tabular}

\begin{tabular}{|c|c|c|}
\hline & Androgenetic Alopecia Group \% & Non-Alopecia Group \% \\
\hline 3 & 10 & 12 \\
\hline $3 \mathrm{a}$ & 2 & 2 \\
\hline $3 \mathrm{v}$ & 0 & 0 \\
\hline 4 & 12 & 0 \\
\hline $4 \mathrm{a}$ & 0 & 0 \\
\hline $5 \mathrm{a}$ & 12 & 14 \\
\hline 6 & 4 & 1 \\
\hline 7 & 2 & 1 \\
\hline \multicolumn{2}{|c|}{ Table 2 Distribution of Samples According to The Grade of Alopecia } \\
\hline
\end{tabular}

Table 2. Distribution of Samples According to The Grade of Alopecia

\begin{tabular}{|c|c|c|c|}
\hline & AGA Group & Non-Alopecia & p Value \\
\hline Central obesity & $12(40 \%)$ & $8(26.6 \%)$ & 0.001 \\
\hline Blood pressure & $10(33.3 \%)$ & $4(13.3 \%)$ & 0.62 \\
\hline triglycerides & $6(20 \%)$ & $8(26.6 \%)$ & 0.005 \\
\hline Reduced HDL & $12(40 \%)$ & $2(6.6 \%)$ & 0.002 \\
\hline Elevated glucose & $10(33.3 \%)$ & $2(6.6 \%)$ & 0.32 \\
\hline \multicolumn{3}{|c|}{ Table 3. Distribution of Components of Metabolic Syndrome } \\
in Both Groups \\
\hline
\end{tabular}

\begin{tabular}{|c|c|c|c|c|}
\hline & $3,3 a, 3 v$ & $\mathbf{4 , 4 a}$ & 5 and above & p Value \\
\hline Central obesity & $6(50 \%)$ & $4(33.3 \%)$ & $4(66.6 \%)$ & 0.011 \\
\hline Raised blood pressure & $6(50 \%)$ & $2(16 \%)$ & $3(25 \%)$ & 0.064 \\
\hline Raised triglycerides & $4(33.3 \%)$ & $6(50 \%)$ & $1(16.6 \%)$ & 0.043 \\
\hline Decreased HDL & $2(16 \%)$ & $2(6 \%)$ & $4(66.6 \%)$ & 0.024 \\
\hline Raised glucose & $3(25 \%)$ & $4(33.3 \%)$ & $2(33.3 \%)$ & 0.031 \\
\hline Metabolic syndrome & $4(33.3 \%)$ & $2(16 \%)$. & $2(33.3 \%)$ & 0.021 \\
\hline Table 4. Association of Grade of Alopecia with Metabolic Syndrome \\
\hline
\end{tabular}

\begin{tabular}{|c|c|c|c|}
\hline & $\begin{array}{c}\text { Androgenetic } \\
\text { Alopecia Group }\end{array}$ & $\begin{array}{c}\text { Non-Alopecia } \\
\text { Group }\end{array}$ & p Value \\
\hline Central obesity & 12 & 8 & 0.237 \\
\hline Raised blood pressure & 10 & 4 & 0.114 \\
\hline Raised triglyceride & 6 & 8 & 0.001 \\
\hline Decreased HDL & 12 & 2 & 0.23 \\
\hline Elevated glucose & 10 & 2 & 0.0337 \\
\hline
\end{tabular}

Table 5. Distribution of Components of Metabolic Syndrome in Androgenic Alopecia

Central obesity and HDL are more associated with AGA group. Triglycerides are more elevated in non-alopecia group.

\begin{tabular}{|c|c|c|c|}
\hline & Grade 3 & Grade 4 & Grade 5 \\
\hline Central obesity & 6 & 4 & 4 \\
\hline $\begin{array}{l}\text { Raised blood } \\
\text { pressure }\end{array}$ & 6 & 2 & 3 \\
\hline Raised triglycerides & 4 & 6 & 1 \\
\hline Decreased HDL & 2 & 2 & 4 \\
\hline Increased glucose & 3 & 4 & 2 \\
\hline Metabolic syndrome & 4 & 2 & 2 \\
\hline \multicolumn{4}{|c|}{ Table 6. Association of Grade of Alopecia with Metabolic Syndrome } \\
\hline \multicolumn{4}{|c|}{$\begin{array}{l}\text { Patients of grade } 3 \text { are mostly having central obesity and raised blood pressure. } \\
\text { Grade } 4 \text { is more associated with decreased HDL and raised glucose. Grade } 5 \text { patients } \\
\text { are having raised glucose mostly. }\end{array}$} \\
\hline
\end{tabular}

\begin{tabular}{|c|c|}
\hline Criteria & Definition \\
\hline Abdominal obesity & $>102 \mathrm{~cm} \mathrm{in} \mathrm{male,},>88 \mathrm{~cm} \mathrm{in} \mathrm{females}$ \\
\hline Triglycerides & $>/=150 \mathrm{mg} / \mathrm{dl}$ \\
\hline High density lipoprotein(HDL) & $<40 \mathrm{mg} / \mathrm{dl}$ \\
\hline High blood pressure & $>/=130 / 85 \mathrm{~mm} \mathrm{hg}$ \\
\hline High fasting glucose & $>/=110 \mathrm{mg} / \mathrm{dl}$ \\
\hline Table 7. Criteria for Metabolic Syndrome \\
\hline
\end{tabular}

\section{METHODS}

This is hospital based descriptive cross-sectional study of two groups androgenetic alopecia group and non-alopecia group, attending the skin outpatient departments between January and March 2018. Samples in the androgenic alopecia group are men in the age 20-45 years with grade 3AGA and above. Samples in the non-alopecia group are men aged 20-45 years having skin disease without androgenic alopecia. Patients taking anti-diabetic medication, antihypertensive medication and hormonal therapy are excluded from the study. Consent is taken from every case. Things noted in the history are age, duration, onset of alopecia. History of smoking, alcohol and family history of androgenetic alopecia is also taken. Blood pressure, triglycerides, high density lipoproteins, triglycerides, waist circumference, fasting blood sugar of patients is taken.

Metabolic syndrome was diagnosed according to new international diabetes federation definition as waist circumference $>102 \mathrm{~cm}$ and any of the two of four factors-

1. Raised triglycerides $>150 \mathrm{mg} / \mathrm{dl}$.

2. Reduced high density lipoprotein $<40 \mathrm{mg} / \mathrm{dl}$.

3. Raised blood pressure systolic $>130$, diastolic $>85$.

4. Raised fasting glucose.

\section{Statistical Analysis}

Analysis is carried out using SPSS version 21 trial. Quantitative variables analysed using students $\mathrm{t}$ test, for qualitative variables chi-square test. Significant $p$ value is $<0.05$. Study includes 30 samples in androgenetic alopecia group and nonalopecia group.

\section{RESULTS}

Metabolic syndrome was seen in 8 out of 30 samples (26\%) in the androgenetic alopecia group, 4 out of 30 samples (13\%) in non-alopecia group, $p$ value is 0.015 . Central obesity and HDL are elevated in most of the samples of androgenetic alopecia group

\section{DISCUSSION}

\section{Androgenetic Alopecia}

Hair loss is a common condition that affects most people in some point of life. It exists as an isolated problem or with other diseases. Many studies have investigated the relationship between androgenetic alopecia and metabolic syndrome and its individual components especially in men. Most of these studies supports the association between androgenetic alopecia and metabolic syndrome.

Androgenetic alopecia represents patterned hair loss primarily affecting scalp hair and is the most common form of hair loss in both men and women. The classic male pattern involves frontal, temporal and vertex areas leaving a rim of hair at the sides and rear of the head. The Hamilton Norwood classification is used to grade androgenetic alopecia in men. Female pattern hair loss presents with diffuse thinning of hair on the crown with sparing of frontal hair line. Female pattern hair loss is graded according to Ludwig's scale. The postulated aetiology leading to androgenetic alopecia (AGA) is complex and multifactorial. Male pattern hair loss appears to be result of both androgen hyperactivity as well as genetic susceptibility.

In AGA the hair cycle sees two major changes, first a decrease in the anagen phase resulting in thinning and shorter 
hairs in a process called follicular miniaturisation. Second, an increase in the number of empty hair follicles. The dominant role of androgens in the process of AGA is well established, in particular the role of dihydrotestosterone (DHT).The finding that AGA develops after puberty when androgen production rises sharply supports this. AGA is not found in castrated men. Dihydrotestosterone is a potent metabolite of testosterone and compared with testosterone has several fold greater affinity to androgen receptors. The enzyme 5 alpha reductase mediates the metabolism of testosterone to dihydrotestosterone. There are two forms of the enzyme type 1 and type 2 . Type 2 plays a dominant role in AGA. DHT subsequently binds to androgen receptors at the hair follicle dermal papillae inhibiting adenyl cyclase and activating genes responsible for the transformation of large follicles in to small ones with shortened anagen phase, causing follicular miniaturization.

\section{Metabolic Syndrome}

Metabolic syndrome, a commonly observed pathology and a global health issue is closely related to skin and thus dermatologists. Metabolic syndrome is a clustering of conditions that occur together and increase the risk of cardiovascular disease, stroke and diabetes. It is largely an effect of an underlying dysfunction of energy, storage and utilisation. The definitions and criteria for metabolic syndrome are constantly revised and threshold vary between different criteria. According to international diabetes federation.

The underlying pathophysiology linking AGA and metabolic syndrome has not been fully established in AGA androgens DHT in particular are main aspects in its pathogenesis. DHT binds to androgen receptors on androgen sensitive follicles and effects follicular miniaturisation. High serum androgen levels also increase the risk of developing hypertension via two mechanisms. Androgen mediated receptors are found in arterial wall endothelium as well increase in serum androgen cause proliferation of smooth muscle cells in vessels, leading to hypertension. The other mechanism concerns increased mineralocorticoid production. The binding of androgens to mineralocorticoid receptors further favours a rise in blood pressure and enhances sensitivity to androgens.

Insulin resistance and excess insulin lower sex hormone binding globulin which leads to high levels of free testosterone and hence AGA. Hyper insulinemia cause increase in local androgen production denovo from cholesterol and through increasing local conversion of testosterone to DHT. The vascular effects of insulin resistance further contribute to this, vasoactive substances that are produced in insulin resistance cause endothelial dysfunction leading to microcirculatory compromise perifollicular vasoconstriction, smooth muscle cell proliferation in the vascular wall. These create microvascular insufficiency and tissue hypoxia at the follicular level and contribute to miniaturisation of hair follicle.

AGA has been reported to be associated with number of diseases including metabolic related diseases such as metabolic syndrome, coronary heart disease, insulin resistance, hypertension, dyslipidaemia and obesity, as well as non-metabolic related diseases such as benign prostatic hyperplasia and prostatic cancer. Cardiovascular disease has become a leading cause of mortality in most countries.
Endogenous androgens have not shown any consistent association with coronary artery disease. Metabolic syndrome has been associated with higher cardiovascular and coronary heart disease mortality even after adjusting for other cardiovascular disease risk factors. The risk increases with number of metabolic syndrome components present.

A few studies have been done on the association of metabolic syndrome with androgenic alopecia. Our study showed increased prevalence of metabolic syndrome in patients with androgenetic alopecia. Our study found significant association with central obesity and HDL levels in cases and triglycerides elevated in controls. Arias Santiago et al in a case control study of 77 participants with androgenic alopecia and 77 controls demonstrated metabolic syndrome in $60 \%$ men with androgenic alopecia.[1] A pro inflammatory state has been suggested as a possible link between pathogenesis of androgenic alopecia and metabolic syndrome. $\mathrm{Su}$ and Chen ${ }^{[2]}$ conducted a population based cross sectional study of 740 patients and found a statistically significant association between androgenic alopecia and components of metabolic syndrome. Insulin parameters are not evaluated in our study. Ekmecki et al, did not find a significant difference in fasting blood glucose in women with androgenic alopecia and controls. ${ }^{[3]}$ Hypertension is not significantly associated with androgenic alopecia when compared to controls in our study. Ahouansou et al found strong association between androgenic alopecia and hypertension in 250 Caucasian men. ${ }^{[4]}$ There are androgen mediated receptors in the arterial wall endothelium. High serum androgen levels in serum contribute to proliferation of smooth muscle cells in vessels and increase the tendency to hypertension. Another explanation for this association is binding of androgens to mineralocorticoid receptors, favouring blood pressure increase or increased peripheral sensitivity to androgens despite their normal circulating levels.

Reduced HDL cholesterol is significantly more common in men with androgenic alopecia compared to controls. Sadigha and Zahed[5] demonstrated lower HDL cholesterol and high triglycerides in men with androgenetic alopecia. Dyslipidaemias may contribute to risk of cardiovascular diseases associated with androgenetic alopecia. Hirsso et al demonstrated higher body mass index and waist circumference in young men with moderate to extensive alopecia compared to men with little or no alopecia.[6]Waist circumference correlates with abdominal fat mass both subcutaneous and intrabdominal. Increased waist circumference in addition to body mass index is a risk factor for mortality.

Several previous studies have investigated the association between metabolic syndrome and androgenetic alopecia. In few studies there were significant differences between cases and controls regarding bodyweight and abdominal circumference. Abdominal fat tissue is associated with serious metabolic disorders such as insulin resistance, hyperinsulinemia, hypertension, increased triglycerides, glucose intolerance and diabetes. Some studies Matilainen et al have pointed out that abdominal fat tissue and waist circumference as independent risk factors for coronary heart disease. [7] In a study by Ola Ahmed et al found fasting blood sugar was high in cases than controls, this result conflicted with the results of Nabaie et al and Acibucu et al.[8] 
In our study, triglycerides were significantly higher in nonalopecia group, whereas HDL values are low in studied cases than in AGA group. Conflictingly Sasmaz et al found significantly higher levels of serum triglycerides in androgenic alopecia group than in non-alopecia group. ${ }^{[9]}$ Androgens were proved to decrease HDL levels in experimental studies. High values of triglycerides and low values of HDL were associated with transition from atheroma to atherosclerosis. Therefore, investigation and control of lipid profiles in patients with androgenetic alopecia may be important to reduce the risk. Contrary to this Guzzo et al found no difference in HDL and triglycerides between androgenetic alopecia patients and normal controls.[10] This inconsistency may be partially attributed to the different target populations studied and to the failure to control other confounding factors, such as family history and smoking status. Conversely YI et al reported nonsignificant association between AGA and metabolic syndrome.[11]

The pathophysiological link between metabolic syndrome and androgenetic alopecia is not yet well understood. Insulin resistance associated with AGA has been previously reported and might contribute to this association. Insulin is found in hair follicles and may play a role in the regulation of androgen metabolism and hair growth cycle, which are relevant to the loss of scalp hair in male pattern baldness. Hyperinsulinemia fasting or post prandial has been shown to be a risk factor for coronary heart disease, accelerates the development of atherosclerosis and prevent atherosclerotic plaque resorption. Gonzale-Gonzale and Mumucoglu et al found relationship between insulin resistance and male pattern baldness.[12]

\section{CONCLUSIONS}

Central obesity and low HDL levels are more commonly associated with AGA group, and elevated triglycerides are mostly seen in non-alopecia group. Distribution according to grades- central obesity is mostly seen in grade 3 AGA. Decreased HDL is mostly seen in grade 4 AGA. Increased glucose levels are mostly seen in grade 5 AGA. Overall metabolic syndrome is more associated with grade 3 AGA. This may contribute to the predisposition of patients with androgenetic alopecia to develop cardiovascular disease. Early screening and interventions for metabolic syndrome and its components in patients with androgenetic alopecia may prevent the development of cardiovascular disease. More prospective studies are required in order to objectively clarify as to whether the increased risk of coronary disease in AGA can be attributed to dyslipidaemia due to androgens or due to insulin resistance. AGA patients should be closely followed up in long term, particularly for coronary heart disease. This raises awareness in susceptible individuals that life style changes like weight control, exercise, inclusion of food products with low glycaemic index in early life can reduce the risk of coronary heart disease.

\section{REFERENCES}

[1] Arias-Santiago S, Gutiérrez-Salmerón MT, CastelloteCaballero L, et al. Androgenetic alopecia and cardiovascular risk factors in men and women: a comparative study. J Am Acad Dermatol 2010;63(3):420-9.

[2] Su LH, Chen TH. Association of androgenetic alopecia with metabolic syndrome in men: a community-based survey. Br J Dermatol 2010;163(2):371-7.

[3] Ekmekci TR, Ucak S, Basat 0, et al. The presence of insulin resistance and comparison of various insulin sensivity indices in women with androgenetic alopecia. Eur J Dermatol 2007;17(1):21-5.

[4] Ahouansou S, Le Toumelin P, Crickx B, et al. Association of androgenetic alopecia and hypertension. Eur J Dermatol 2007;17(3):220-2.

[5] Sadighha A, Zahed GM. Evaluation of lipid levels in androgenetic alopecia in comparison with control group. J Eur Acad Dermatol Venereol 2009;23(1):80-1.

[6] Hirsso P, Rajala U, Hiltunen L, et al. Obesity and lowgrade inflammation among young Finnish men with early-onset alopecia. Dermatology 2007;214(2):125-9.

[7] Matilainen V, Koskela P, Keinanen-Kiukaanniemi S. Early androgenetic alopecia as a marker of insulin resistance. Lancet 2000;356(9236):1165-6.

[8] Nabaie L, Kavand S, Robati RM, et al. Androgenic alopecia and insulin resistance: are they really related? Clin Exp Dermatol 2009;34(6):694-7.

[9] Sasmaz S, Senol M, Ozcan A, et al. The risk of coronary heart disease in men with androgenetic alopecia. J Eur Acad Dermatol Venereol 1999;12(2):123-5.

[10] Guzzo CA, Margolis DJ, Johnson J. Lipid profiles, alopecia and coronary disease: any relationship? Dermatol Surg 1996;22(5):481.

[11] Yi SM, Son SW, Lee KG, et al. Gender-specific association of androgenetic alopecia with metabolic syndrome in a middle-aged Korean population. $\mathrm{Br} \mathrm{J}$ Dermatol 2012;167(2):306-13.

[12] Mumcuoglu C, Ekmekci TR, Ucak S. The investigation of insulin resistance and metabolic syndrome in male patients with early-onset androgenetic alopecia. Eur J Dermatol 2011;21(1):79-82. 